\title{
Light Transport in Soft Tissue Based on Simplified Spherical Harmonics Approximation to Radiative Transport Equation
}

\author{
Michael K. Chu' ${ }^{1}$, Alexander D. Klose ${ }^{2}$ and Hamid Dehghani ${ }^{1}$ \\ ${ }^{I}$ School of Physics, University of Exeter, Stocker Road, Exeter, EX4 4QL, UK \\ ${ }^{2}$ Department of Radiology, Columbia University, New York, NY 10032, USA \\ mc283@ex.ac.uk
}

\begin{abstract}
The simplified spherical harmonics $\left(\mathrm{SP}_{\mathrm{n}}\right)$ approximation is implemented into the NIRFAST package. Preliminary studies are presented on a small $10 \mathrm{~mm} \times 10 \mathrm{~mm}$ geometry and qualitative results are shown for $\mathrm{SP}_{1}, \mathrm{SP}_{3}$ and $\mathrm{SP}_{5}$. Further work is underway to quantitatively test the accuracy of the $\mathrm{SP}_{\mathrm{N}}$ models using Monte Carlo data as a benchmark.

(C)2007 Optical Society of America
\end{abstract}

OCIS codes: (170.3660) Light propagation in tissues; (170.6960) Tomography

\section{Introduction}

Optical Tomography is a non-invasive medical imaging technique in which near-infrared (NIR) light is used to obtain information about the optical properties of biological tissues. An experimental setup would involve light being guided to the surface of a subject using fibre optics. A series of detector fibres are then used to collect the transilluminated light [1]. In order to create a meaningful image from the detected light, an accurate model of light propagation in tissue is required.

It has been well established that an accurate model of photon transport can be provided by the radiative transfer equation (RTE). Due to the computational expense of solving the RTE, however, the diffusion approximation is commonly used. The diffusion approximation is based on the assumption that the distribution of light within the subject is diffuse and therefore the scattering in the region of interest is dominant over absorption. The approximation also requires that the light sources and detectors of the imaging system are separated by more than a few scattering distances in order to avoid a forwardly biased light distribution.

The diffusion approximation proves to be adequate when applied to large, highly scattering media such as in female breast imaging [2,3]. One of the other major applications of optical tomography, however, is in the imaging of small animals in order to develop models for disease such as cerebral ischemia or cancer [4]. Due to the small geometries encountered in small animal imaging, the seperation between the light sources and detectors is small, resulting in a forwardly biased distribution of light. This means that the diffusion approximation is insufficient and a more suitable model is required.

\section{Simplified Spherical Harmonics}

The simplified spherical harmonics $\left(\mathrm{SP}_{\mathrm{N}}\right)$ equations provide an approximation to the RTE that can overcome some of the limitations of the diffusion approximation. They also have the advantage of being considerably less computationally demanding than other high order approximations such as the discrete ordinates method or the standard spherical harmonics method. A full 3 dimensional problem can be solved up to the order $\mathrm{N}=7$ with a set up just four coupled equations. As derived by Klose et al [5], the four coupled equations constituting the $\mathrm{SP}_{7}$ approximation are:

$$
\begin{aligned}
& -\nabla \cdot \frac{1}{3 \mu_{a 1}} \nabla \varphi_{1}+\mu_{a} \varphi_{1}=Q+\left(\frac{2}{3} \mu_{a}\right) \varphi_{2}+\left(\frac{8}{15} \mu_{a}\right) \varphi_{3}+\left(\frac{16}{35} \mu_{a}\right) \varphi_{4} \\
& -\nabla \cdot \frac{1}{7 \mu_{a 3}} \nabla \varphi_{2}+\left(\frac{4}{9} \mu_{a}+\frac{5}{9} \mu_{a 2}\right) \varphi_{2}=-\frac{2}{3} Q+\left(\frac{2}{3} \mu_{a}\right) \varphi_{1}+\left(\frac{16}{45} \mu_{a}+\frac{4}{9} \mu_{a 2}\right) \varphi_{3}-\left(\frac{32}{105} \mu_{a}+\frac{8}{21} \mu_{a 2}\right) \varphi_{4} \\
& -\nabla \cdot \frac{1}{11 \mu_{a 5}} \nabla \varphi_{3}+\left(\frac{64}{225} \mu_{a}+\frac{16}{45} \mu_{a 2}+\frac{9}{25} \mu_{a 4}\right) \varphi_{3}=\frac{8}{15} Q-\left(\frac{8}{15} \mu_{a}\right) \varphi_{1} \\
& +\left(\frac{16}{45} \mu_{a}+\frac{4}{9} \mu_{a 2}\right) \varphi_{2}+\left(\frac{128}{525} \mu_{a}+\frac{32}{105} \mu_{a 2}+\frac{54}{175} \mu_{a 4}\right) \varphi_{4}
\end{aligned}
$$




\section{BSuE42.pdf}

$$
\begin{aligned}
& -\nabla \cdot \frac{1}{15 \mu_{a 7}} \nabla \varphi_{4}+\left(\frac{256}{1225} \mu_{a}+\frac{64}{245} \mu_{a 2}+\frac{324}{1225} \mu_{a 4}+\frac{13}{49} \mu_{a 6}\right) \varphi_{4}=-\frac{16}{35} Q+\left(\frac{16}{35} \mu_{a}\right) \varphi_{1} \\
& +\left(\frac{32}{105} \mu_{a}+\frac{8}{21} \mu_{a 2}\right) \varphi_{2}-\left(\frac{128}{525} \mu_{a}+\frac{32}{105} \mu_{a 2}+\frac{54}{175} \mu_{a 4}\right) \varphi_{3}
\end{aligned}
$$

where

$$
\begin{aligned}
& \varphi_{1}=\phi_{0}+2 \phi_{2} \\
& \varphi_{2}=3 \phi_{2}+4 \phi_{4} \\
& \varphi_{3}=5 \phi_{4}+6 \phi_{6} \\
& \varphi_{4}=7 \phi_{6} \\
& \mu_{a n}=\mu_{t}-\mu_{s} g^{n}
\end{aligned}
$$

These equations can be easily reduced to obtain the lower order approximations. The $\mathrm{SP}_{5}$ equations are obtained by setting $\phi_{6}=0$ and solving equations (1)-(3). The $\mathrm{SP}_{3}$ equations are obtained by setting $\phi_{6}=\phi_{4}=0$ and solving equations (1) \& (2) and the $\mathrm{SP}_{1}$ equation reduces explicitly to the diffusion equation by setting $\phi_{6}=\phi_{4}=\phi_{2}=0$ and solving equation (1).

\section{Method}

The simplified spherical harmonics approximations $\left(\mathrm{SP}_{1}, \mathrm{SP}_{3}, \mathrm{SP}_{5}\right)$ were implemented into an existing finite-element based diffusion solver (NIRFAST). Preliminary studies are shown on a square model of $10 \mathrm{~mm} \times 10 \mathrm{~mm}$ with a node spacing of $0.08 \mathrm{~mm}$, Figure 1. The optical properties of the domain were defined as $\mu_{a}=0.001 \mathrm{~mm}^{-1}, \mu_{s}=1 \mathrm{~mm}^{-1}$, $g=0, n=1$. A single light source, with a width of $2 \mathrm{~mm}$, was applied at one edge of the domain.

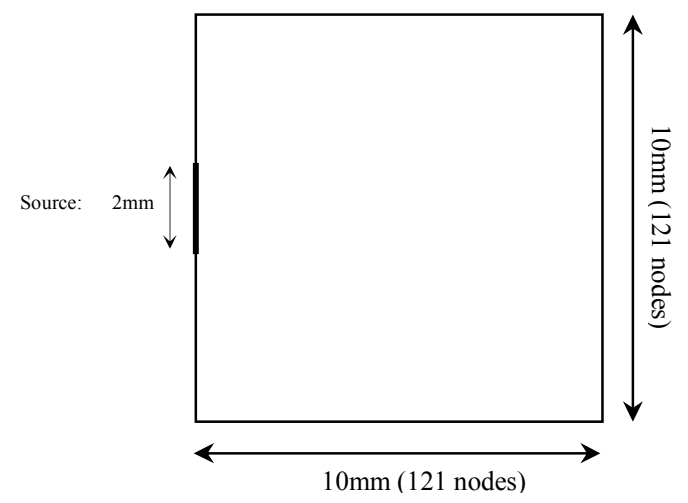

Figure 1: Schematic of the model used in preliminary studies

\section{Results}

Solutions for the $\mathrm{SP}_{1} \mathrm{SP}_{3}$ and $\mathrm{SP}_{5}$ approximations are shown in Figure 2, together with the percentage difference calculated between each spherical expansion. As seen the results appear to correlate, with the main source of difference occurring in the regions near the source. The percentage difference between $\mathrm{SP}_{1}$ and $\mathrm{SP}_{3}$ is approximately $34 \%$ with the largest difference seen near the source. At higher order, the percentage difference at the source (between $\mathrm{SP}_{3}$ and $\mathrm{SP}_{5}$ ) is $24 \%$. Additionally, differences at the external boundaries are also observed, indicating that the measured boundary data are also affected by the size of the domain.

\section{Discussion}

The $\mathrm{SP}_{\mathrm{N}}$ equations have been implimented within NIRFAST, for $\mathrm{N}=1,3$ and 5 and preliminary results have been presented. A model for $\mathrm{SP}_{7}$ is currently being developed. Further work is necessary in order to verify the accuracy 


\section{BSuE42.pdf}

of the $\mathrm{SP}_{\mathrm{N}}$ approximations. Comparitive studies will be made using Monte Carlo data as a benchmark and the accuracy of the models will be quantified. Work is also being done to improve the computational efficiency of the models.

$\mathrm{SP}_{1}$
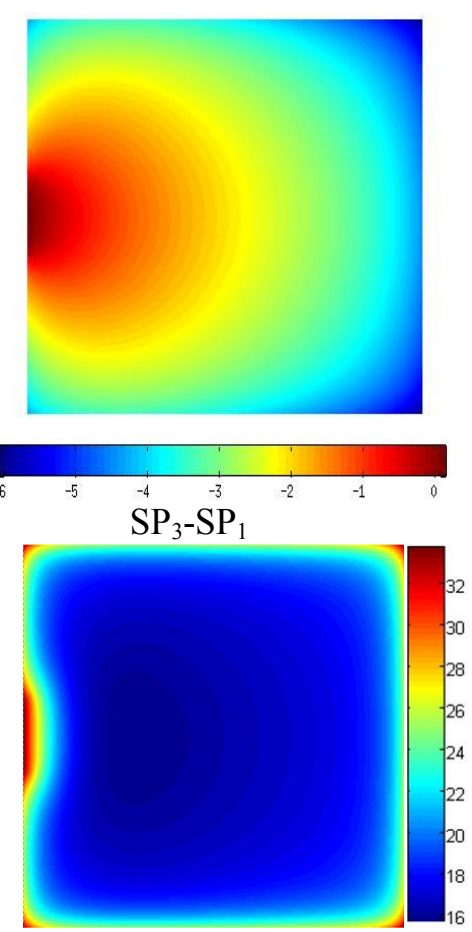

$\mathrm{SP}_{3}$
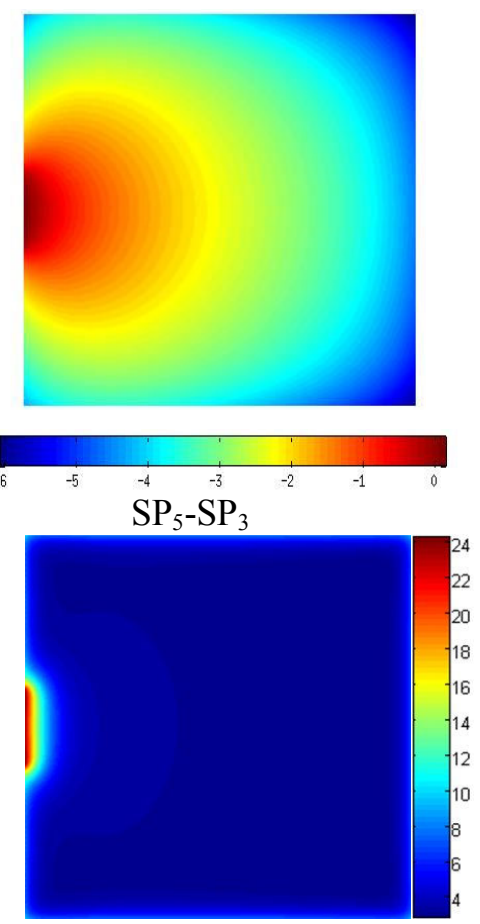

$\mathrm{SP}_{5}$
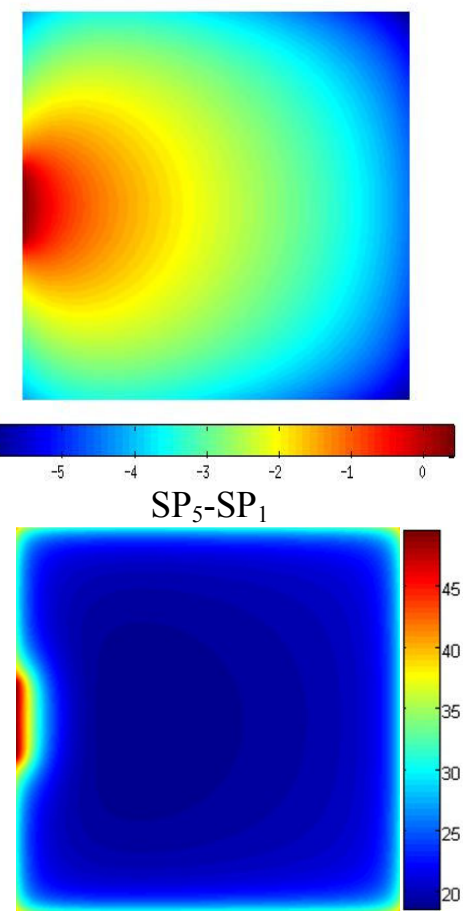

Figure 2: The solution calculates for SP1, SP3 and SP3 (top row) for model shown in Figure 1, and the percentage difference between the solution at each spherical harmonics (bottom row)

\section{Acknowledgements}

This work has been sponsored by EPSRC, UK.

\section{References}

1. $\quad$ Arridge, S.R., Optical tomography in medical imaging. Inverse Problems, 1999. 15(2): p. R41-R93.

2. Dehghani, H., et al., Multiwavelength three-dimensional near-infrared tomography of the breast: initial simulation, phantom, and clinical results. Applied Optics, 2003. 42(1): p. 135-145.

3. Carp, S.A., et al., Compression-induced changes in the physiological state of the breast as observed through frequency domain photon migration measurements. Journal of Biomedical Optics, 2006. 11(6).

4. Hielscher, A., Optical tomographic imaging of small animals. Medical Physics, 2005. 32(6): p. $2096-2096$.

5. Klose, A.D. and E.W. Larsen, Light transport in biological tissue based on the simplified spherical harmonics equations. Journal of Computational Physics, 2006. 220(1): p. 441-470. 